\title{
In situ sulfidation of Pd/C: A straightforward method for chemoselective conjugate reduction by continuous hydrogenation
}

\author{
Jonathan C. Moore, ${ }^{a}$ Rowena A. Howie, ${ }^{a}$ Samuel L. Bourne ${ }^{b}$ Gareth N. Jenkins, ${ }^{b}$ Peter Licence, ${ }^{a}$ \\ Martyn Poliakoff ${ }^{a}$ and Michael W. George ${ }^{* a, c}$ \\ a) School of Chemistry, University of Nottingham, University Park, Nottingham, NG7 2RD, UK. \\ E-mail: mike.george@nottingham.ac.uk \\ b) Arcinova, Taylor Drive, Alnwick, Northumberland, NE66 2DH, UK. \\ c) Department of Chemical and Environmental Engineering, University of Nottingham Ningbo China, 199 Taikang East Road, Ningbo 315100 , \\ China.
}

Supporting Information. Experimental procedures, compound characterisation and GreenMotion ${ }^{\mathrm{TM}}$ analysis.

\begin{abstract}
A method has been developed for the in situ sulfidation of $\mathrm{Pd} / \mathrm{C}$ under continuous flow. The approach provides a cheap, sustainable and operationally convenient method for chemoselective conjugate reduction by continuous hydrogenation. High conversions and excellent selectivities were obtained for olefin reduction in $\alpha / \beta$-unsaturated carbonyl compounds in the presence of hydrogenatively sensitive functionalities. The methodology was analysed with a green metric system to highlight the sustainability features of the process.
\end{abstract}

\section{Key Words}

Hydrogenation; Continuous-Flow; Chemoselective; Conjugate-Reduction; Chalcones.

\section{Synopsis}

$\alpha / \beta$-Unsaturated carbonyl compounds are chemoselectively hydrogenated in continuous-flow via catalyst modification with diphenyl sulfide.

\section{Introduction}

Over the past few decades there has been increasing pressure on pharmaceutical manufacturers to apply green metrics and improve efficiencies across the spectrum of chemical processes that they employ. ${ }^{1-3}$ Consequently, an emphasis has been placed on the implementation of continuous manufacturing. ${ }^{4-9}$ As a result, many enabling technologies have become available to drive this new agenda. ${ }^{10-33}$ Catalytic heterogeneous hydrogenation is a key process technology that has benefitted from the growing popularity and commercial availability of miniaturised continuous hydrogenation reactors. Consequently, there is a growing literature precedent describing continuous hydrogenation processes. ${ }^{34-38}$ Previous work from our group on continuous hydrogenation has largely focused on the use of supercritical fluids $(\mathrm{SCFs})^{39,40}$ and includes the large-scale hydrogenation of isophorone ${ }^{41}$ and the reductive cyclisation of levulinic acid to $\mathrm{X}$-valerolactone. ${ }^{42}$ With the increasing expertise and knowledge, contract development and manufacturing organisations (CDMOs), which have historically taken a risk adverse approach to the adoption of new technologies, are turning to continuous manufacturing and applying green metrics to improve their own processes. As part of our ongoing drive towards greener continuous process improvement we were keen to investigate the reduction of Michael acceptors via continuous hydrogenation; in particular, a process relevant to our collaboration with industrial partners - the conjugate reduction of chalcones to dihydrochalcones. Dihydrochalcones are members of the flavonoid family and are widely distributed throughout the plant kingdom. ${ }^{43}$ They exhibit a variety of biological properties including: antimicrobial, anti-inflammatory, antioxidant, antiplasmodial and antiviral activities and make up the core of many active pharmaceutical ingredients (Figure 1). ${ }^{44-46}$ The most appealing method to access dihydrochalcones is conjugate reduction of chalcones, which can themselves be easily synthesised via the Claisen-Schmidt condensation between benzaldehyde and acetophenone derivatives. ${ }^{47}$ The conjugate reduction of chalcones presents a challenge however, as the benzylic nature of the ketone functionality renders these substrates highly susceptible to over-reduction. This presents a significant challenge, particularly on large scale when employing traditional batch manufacturing approaches. Indeed, the difficulties associated with the chemoselectivity of this transformation are well documented. ${ }^{48-50}$ 


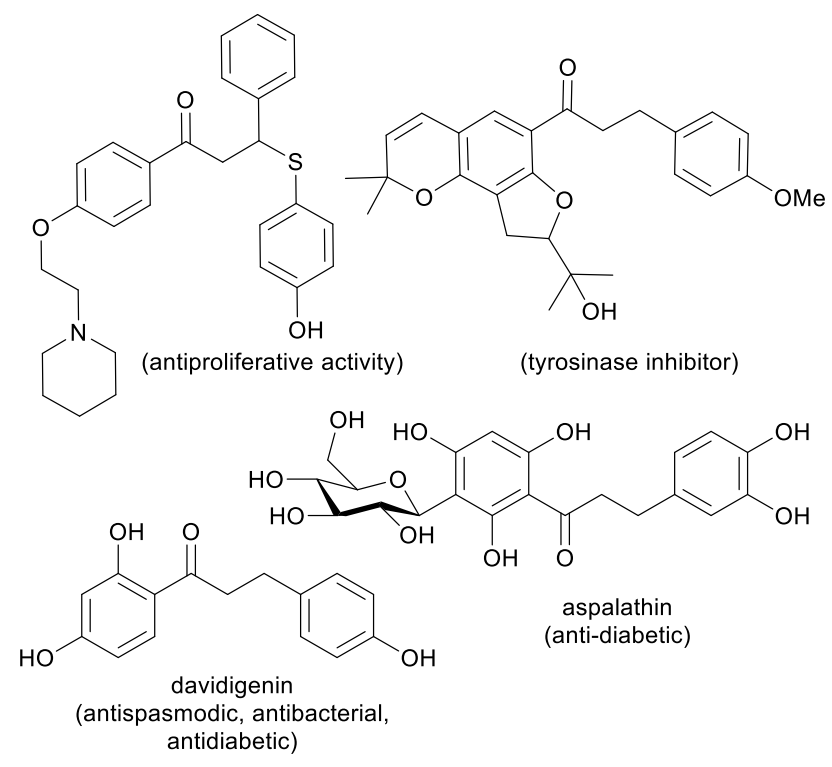

Fig. 1. Examples of biologically active molecules containing the dihydrochalcone scaffold.

Whilst a number of methods have been reported for chemoselective conjugate reduction via homogenous catalysis, few of these are currently amenable to a continuous-flow protocol. For example, a number of approaches have been developed that utilise bisphosphine ligated $\mathrm{CuH}$ catalysts. ${ }^{51,52}$ These highly carbophilic reducing agents have seen great utility in the asymmetric conjugate reduction of a range of $\alpha / \beta$-unsaturated carbonyl compounds including ketones, ${ }^{53}$ esters $^{54}$ and carboxylic acids. ${ }^{55}$ To the best of our knowledge however, no continuous-flow procedure has been reported that capitalises on this approach. Studies by Winterbottom and co-workers on the heterogeneous conjugate reduction of cinnamaldehyde focused on the effect of Fe and $\mathrm{K}$ salts and quinolone as additives on the chemoselectivity of the process. ${ }^{56}$ The most selective conditions utilised potassium acetate as the additive and yielded $85 \%$ selectivity for conjugate reduction. Microfluidic enone reductions have been reported by Kobayashi and co-workers ${ }^{57}$ and a continuous-flow procedure that utilises maghemite-Pd nanocomposites has also been developed for the conjugate reduction of $\alpha / \beta$-unsaturated esters. ${ }^{58} \mathrm{An}$ asymmetric conjugate reduction, with continuous recovery of the chiral auxiliary has been recently reported where $\alpha / \beta$-unsaturated amides are generated in situ from the corresponding acyl chloride and a chiral amine ${ }^{59} \mathrm{~A}$ continuous flow procedure for the ThalesNano $\mathrm{H}$-Cube ${ }^{\mathrm{TM}}$ was reported by Bäckvall and co-workers, where nanopalladium supported on amino-functionalised mesocellular foam (Pd0-AmP-MCF) was employed. ${ }^{60}$ Whilst this was shown to be an effective method, the fabrication of bespoke catalyst cartridges was required via collaboration with ThalesNano. Furthermore, the selectivity was somewhat limited; in the case of chalcone (1), a 9:1 ratio of ketone:alcohol was produced. The deuteration of chalcones has also been performed in an $\mathrm{H}$-Cube ${ }^{\mathrm{TM}}$ reactor, where $\mathrm{D}_{2}$ was generated by the electrolysis of $\mathrm{D}_{2} \mathrm{O} .{ }^{61}$

Our strategy for developing a chemoselective conjugate reduction by continuous hydrogenation was inspired by a series of reports by Sajiki and co-workers, where various sulfur species were employed as additives in Pd/C catalysed hydrogenation in batch format. ${ }^{62-64}$ Crucially for this study, it was shown that diphenyl sulfide imparts excellent selectivity when employed as an additive in the $\mathrm{Pd} / \mathrm{C}$ catalysed conjugate reduction of chalcone (1). Sulfur species are well known to poison many metal catalysts, hence the advent of ultralow sulfur gasoline and diesel, which serves to prolong the lifetime of catalytic-converters in automotive exhaust streams. On the other hand, there are in fact many cases where sulfur additives can act as activity promotors and/or selectivity modifiers via modulation of the electronic environment and/or the orientation of catalytic metal centres. ${ }^{65}$ The interplay between these distinct modes of action can allow for precise tuning of catalyst activity and sulfur additives have seen great utility in a range of chemoselective hydrogenations. ${ }^{66}$ Examples include the conjugate reduction of enones, ${ }^{62}$ hydrodeoxygenation of fructose, ${ }^{67}$ the partial hydrogenation of alkynes, ${ }^{68,69}$ nitro-reduction in the presence of alkenes ${ }^{70}$ and alkene reduction in the presence of aryl chlorides, nitriles, benzyl esters and $\mathrm{N}$-Cbz protecting groups. ${ }^{63}$ We envisaged that if this powerful approach could be incorporated into a flow procedure, it would constitute a highly convenient method for conjugate reduction by continuous hydrogenation. 


\section{Results and Discussion}

Initially, the reduction of chalcone (1) was investigated under a continuous flow protocol in the absence of an additive. Reactions were performed using a ThalesNano $\mathrm{H}-\mathrm{Cube}^{\mathrm{TM}}$ hydrogenation reactor, which generates hydrogen on demand by the electrolysis of water. At $3 \mathrm{~mL} \mathrm{~min}{ }^{-1}$ flow rate, high selectivity for the desired ketone 1a was observed however the reaction proceeded only to $16 \%$ conversion (Table 1, Entry 1 ). Increasing the residence time by reducing the flow rate to $1 \mathrm{~mL} \mathrm{~min}^{-1}$ had only a minimal effect on both conversion and selectivity. (Table 1, Entry 2). A further reduction of the flow rate to $0.5 \mathrm{~mL} \mathrm{~min} \mathrm{~m}^{-1}$ led to an improved conversion (46\%) but in this case a mixture of the ketone 1a and the alcohol $\mathbf{1 b}$ was obtained (Table 1, Entry 3). Similarly, increasing the temperature to $50{ }^{\circ} \mathrm{C}$ gave an improved conversion, but at the cost of selectivity (Table 1, Entry 4). It was apparent at this early stage in the investigation that precise control of the reaction parameters alone would be unlikely to provide satisfactory selectivity in the reduction. This led us to investigate the use of an additive and in light of the excellent results reported for the corresponding batch process (vide supra), we selected diphenyl sulfide.

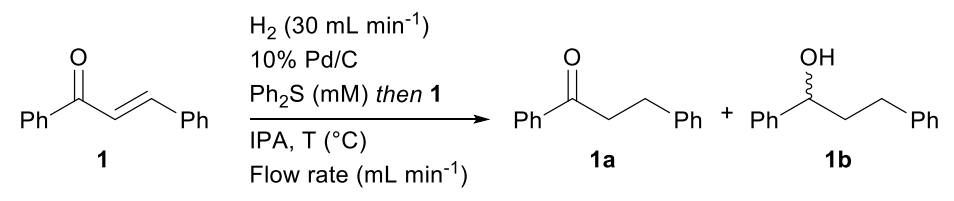

\begin{tabular}{|c|c|c|c|c|c|c|}
\hline \multirow[t]{2}{*}{ Entry } & \multirow[t]{2}{*}{$\mathrm{T}\left({ }^{\circ} \mathrm{C}\right)$} & \multirow{2}{*}{$\begin{array}{l}\text { Flow Rate } \\
\left.(\mathrm{mL} \mathrm{min})^{-1}\right)\end{array}$} & \multirow{2}{*}{$\begin{array}{l}\mathrm{Ph}_{2} \mathrm{~S} \\
(\mathrm{mM})\end{array}$} & \multicolumn{2}{|c|}{ Product Ratio [\%] } & \multirow{2}{*}{$\begin{array}{c}\text { Total } \\
\text { Conversion } \\
{[\%]}\end{array}$} \\
\hline & & & & $1 a$ & $1 \mathrm{~b}$ & \\
\hline 1 & 20 & 3.0 & - & $>95 \%$ & $<5 \%$ & $16 \%$ \\
\hline 2 & 20 & 1.0 & - & $>95 \%$ & $<5 \%$ & $22 \%$ \\
\hline 3 & 20 & 0.5 & - & $83 \%$ & $17 \%$ & $46 \%$ \\
\hline 4 & 50 & 0.5 & - & $74 \%$ & $26 \%$ & $88 \%$ \\
\hline 5 & 50 & 0.5 & 2.5 & $>95 \%$ & $<5 \%$ & $36 \%$ \\
\hline 6 & 100 & 0.5 & 2.5 & $>95 \%$ & $<5 \%$ & $86 \%$ \\
\hline 7 & 100 & 0.5 & 0.5 & $63 \%$ & $37 \%$ & $>95 \%$ \\
\hline 8 & 100 & 0.5 & 1.25 & $>95 \%$ & $<5 \%$ & $>95 \%$ \\
\hline
\end{tabular}

Table 1. Optimisation of reaction conditions. All reactions were performed on $0.5 \mathrm{mmol}$ scale. Conversions and product ratios were determined by ${ }^{1} \mathrm{H}$ NMR analysis.

One challenge in incorporating a potential catalyst poison into a continuous flow protocol is that continuous pumping of the additive across the catalyst bed can result in a cumulative suppression of catalyst activity, making it challenging to maintain steady-state conditions in the reactor. Following preliminary investigations, we developed a procedure that consisted of pre-treatment of the catalyst bed for 20 minutes with a solution of diphenyl sulfide at $0.5 \mathrm{~mL} \mathrm{~min}^{-1} \mathrm{flow}$ rate (Figure 2). Following this period of conditioning, the starting material was processed as an additive-free solution under the same reaction conditions (Table 1). When the sulfide was employed at $2.5 \mathrm{mM}$, excellent selectivity (>95\%) for the desired ketone 1a was observed, albeit with only moderate conversion (36\%, Table 1, Entry 5). The conversion was improved to $86 \%$, without a deleterious effect on the selectivity, by increasing the temperature to $100{ }^{\circ} \mathrm{C}($ Table 1 , Entry 6). Full conversion of the starting material was observed when the sulfide was employed at the lower concentration of $0.5 \mathrm{mM}$ but under these conditions the selectivity was poor (Table 1, Entry 7). This implies that alongside improving the selectivity, the incorporation of diphenyl sulfide leads to a suppression in the desired olefin hydrogenation. The optimal conditions were found when the additive was employed at $1.25 \mathrm{mM}$ and the substrate was processed at $100{ }^{\circ} \mathrm{C}$ (Table 1, Entry 8). Under these conditions, full conversion of the starting material and $>95 \%$ selectivity for the targeted ketone 1a was observed. 


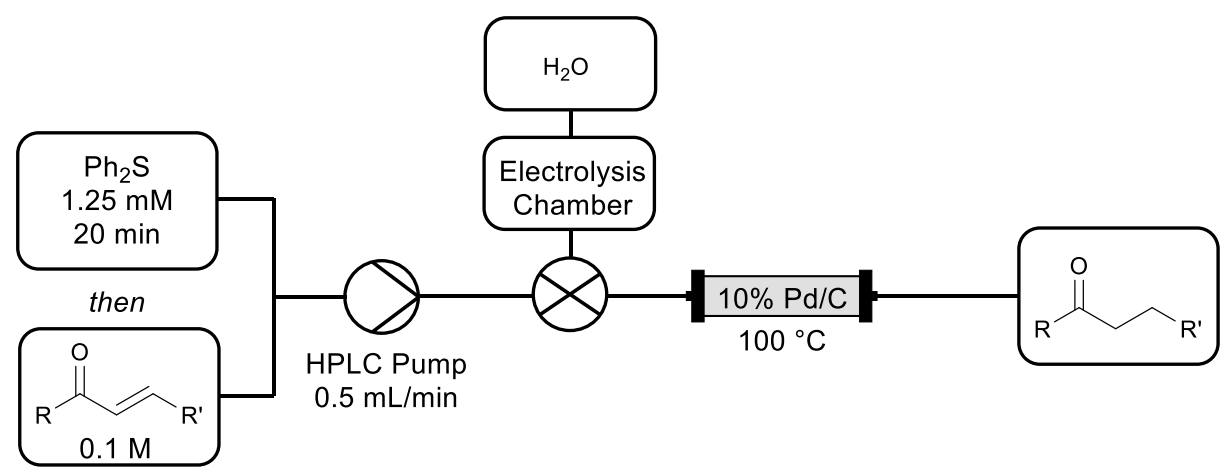

Fig. 2. Flow schematic. Hydrogen gas is generated by electrolysis of water and then mixed with the liquid stream before being passed through the catalyst cartridge.

With the optimised conditions in hand, the substrate scope of the methodology was investigated. Initially a series of chalcone derivatives were targeted in order to establish the effects of electron-donating and -withdrawing substituents at various positions on the phenyl rings. In general, high conversions and selectivities were obtained across the series (Table 2).

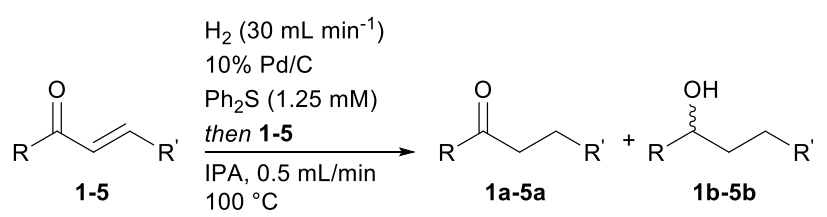

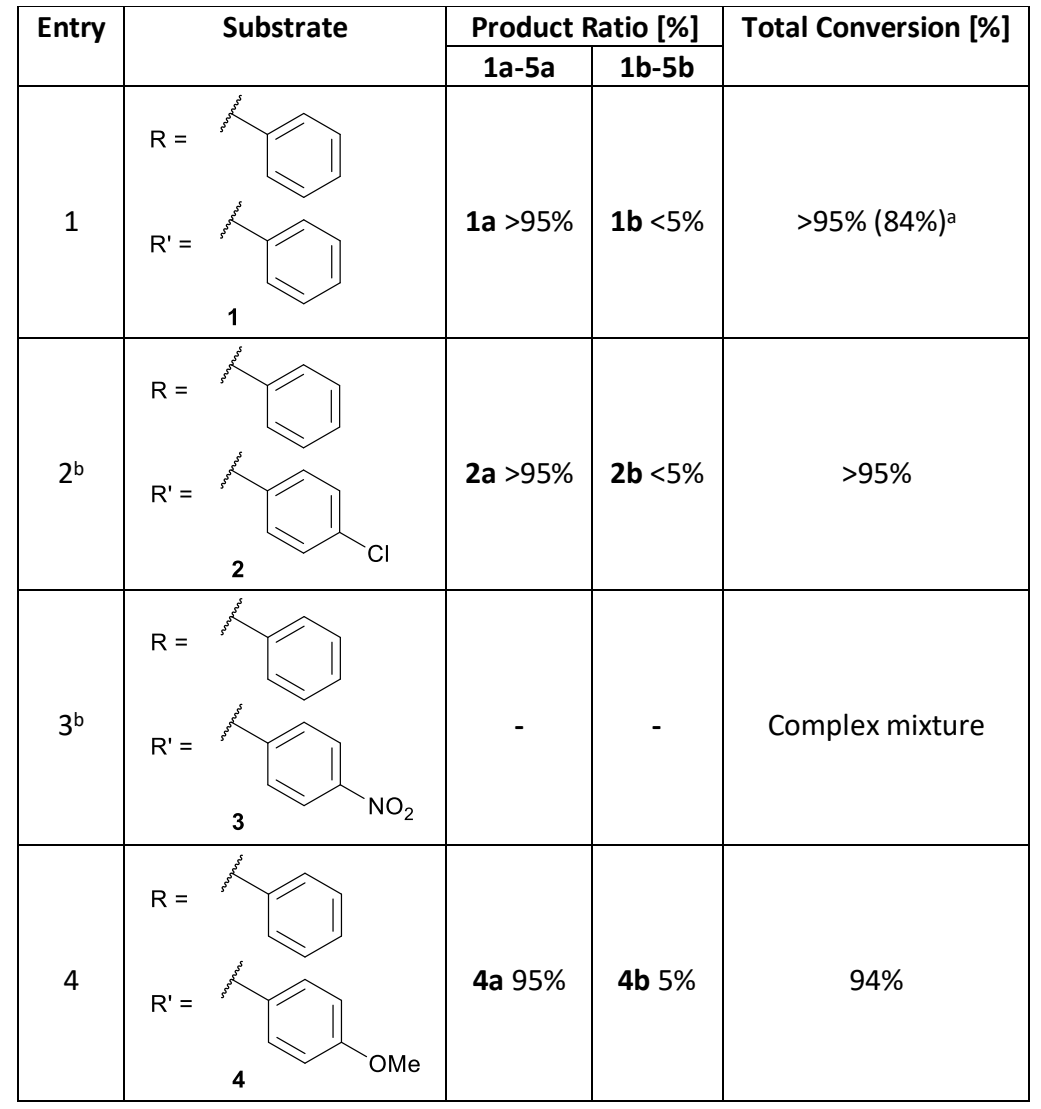




\begin{tabular}{|l|l|l|l|l|}
\hline 5 & & $5 \mathbf{a}>95 \%$ & $5 \mathbf{b}<5 \%$ & \\
\hline
\end{tabular}

Table 2. Substrate scope for the conjugate reduction of chalcones $\mathbf{1 - 5}$. Conversions and product ratios were determined by ${ }^{1} \mathrm{H}$ NMR

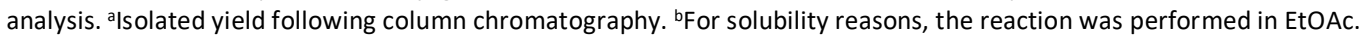

The chemoselective conjugate reduction of the $p$-chloro derivative $\mathbf{2}$ demonstrates that the hydrogenatively unstable aryl chloride functionality is tolerated under the reaction conditions; the hydrodehalogenated species was not observed. In the case of the $p$-nitro derivative $\mathbf{3}$, a complex mixture of products was obtained, which is consistent with the results obtained from the batch procedure reported by Sajiki and co-workers. ${ }^{63}$ Electron-donating substituents were well tolerated, with the $p$-methoxy derivative 4 yielding the targeted dihydrochalcone $4 a$ in $94 \%$ conversion and with $>95 \%$ selectivity. We next turned our attention to the conjugate reduction of isoliquiritigenin (5), a commonly occurring natural product with a range of useful pharmacological properties that include anti-inflammatory and anti-cancer activity. ${ }^{71}$ This compound was transformed into the respective dihydrochalcone, davidigenin (5a) (Figure 1), which is itself a naturally occurring anti-spasmodic that exhibits antidiabetic and weak antibacteral activity. ${ }^{72}$

Next, the scope of the optimised hydrogenation conditions was investigated with a range of non-chalcone derivatives (Table 3).

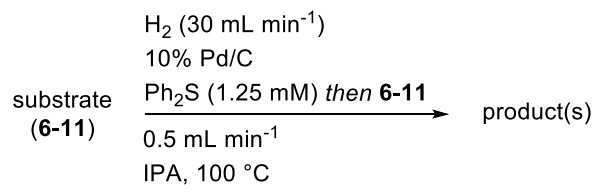

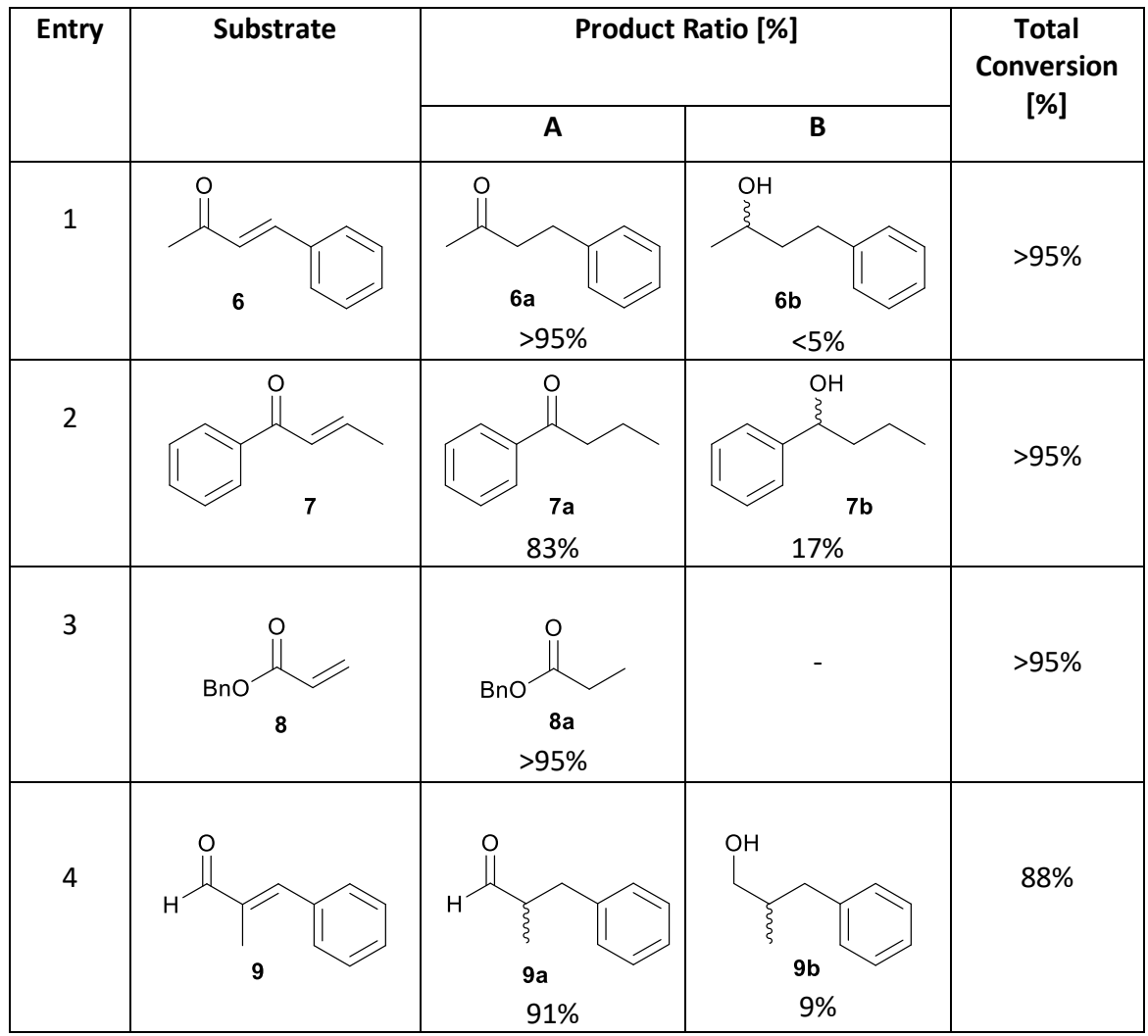




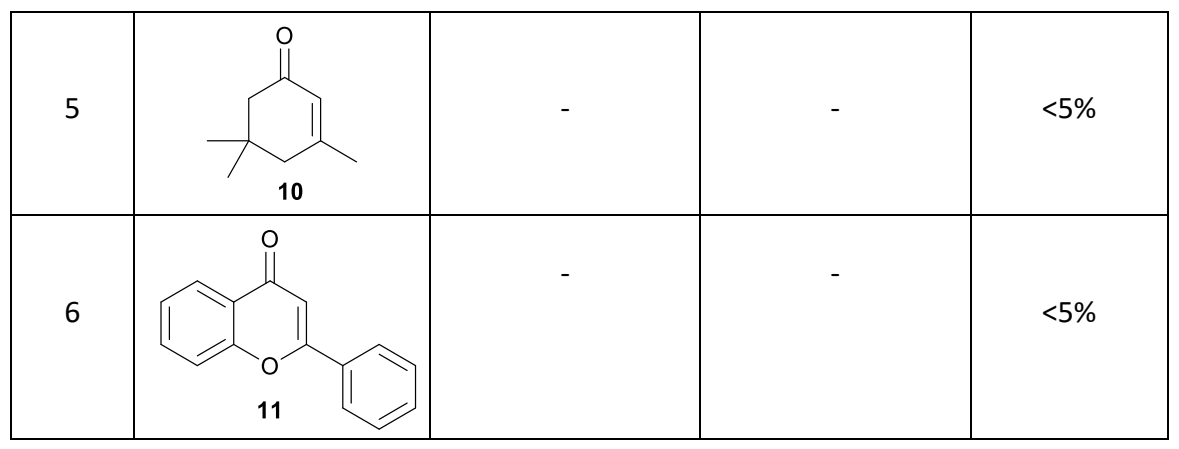

Table 3. Additional substrate scope for conjugate reduction of Michael Acceptors. Conversions and product ratios were determined by ${ }^{1} \mathrm{H}$ NMR analysis.

Pleasingly, the 1- and 4-phenyl butenones $\mathbf{6}$ and $\mathbf{7}$ were each hydrogenated to full conversion and with good selectivity ( $83 \%$ and $>95 \%$, respectively) (Table 3, Entries 1-2). The methodology can also be extended to $\alpha / \beta$-unsaturated esters, as evidenced by the chemoselective hydrogenation of benzyl acrylate (8) (Table 3, Entry 3). This example also demonstrates that the sensitive benzyl ester functionality remains intact under the reaction conditions, with no sign of debenzylation occurring. When the methodology was applied to 2-methyl-cinnamaldehyde (9), the reaction reached $88 \%$ conversion and the targeted aldehyde $9 \mathrm{a}$ was obtained with $91 \%$ selectivity over the corresponding alcohol $9 \mathrm{~b}$. A limit to the methodology was found when the conditions were applied to the hydrogenation of less activated enones; in the cases of isophorone (10) and flavone (11) the major component recovered was starting material (Table 3, Entries 5-6).

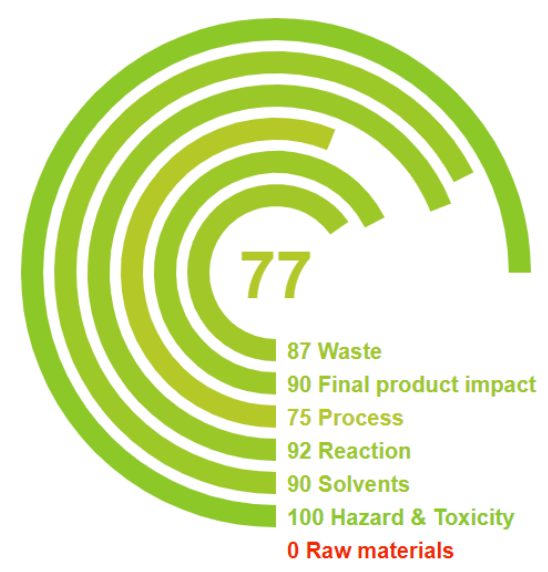

Fig. 3. Analysis of the sustainability profile of the process using the metric tool Green Motion ${ }^{\mathrm{TM}}$.

Using the metric tool Green Motion ${ }^{\mathrm{TM}}$, we have evaluated the methodology with respect to sustainability (Figure 3). ${ }^{73}$ This metric combines the twelve principles of green chemistry ${ }^{74}$ with a penalty point system; the lower the impact to the environment, the higher the rating (100 being ideal). The process adheres well to the principles of green chemistry and therefore scores highly, with key sustainability features including:

- High selectivity, therefore reduced waste - low E-factor (Principle 1) $)^{75}$

- Hydrogenation with $\mathrm{H}_{2}$ - high atom economy (Principle 2) ${ }^{76}$

- Low toxicity of reagents (Principle 3)

- $\quad$ Catalytic quantity of the auxillary diphenyl sulfide (Principle 5)

- Operation at atmospheric pressure and, despite heating, no cooling is required (Principle 6)

- Use of a renewable solvent (Principle 7)

- Catalytic reagents (Principle 9)

- $\quad$ No storage of $\mathrm{H}_{2}$ - reduced chance of accidents (Principle 12) 
A detailed description of the process involved in the GreenMotion ${ }^{\mathrm{TM}}$ assessment is available in the Supporting Information. It should be noted that the low score obtained for "Raw materials" is inherent, as the starting material, chalcone (1), is of synthetic origin. Overall the methodology scored highly at 77/100 which according to the guidelines laid out within the Green Motion ${ }^{\mathrm{TM}}$ metric $^{73}$ validates that the process can be considered green.

\section{Conclusions}

In conclusion, a procedure has been developed for the in situ sulfidation of $\mathrm{Pd} / \mathrm{C}$ in continuous flow. The approach provides a straightforward and sustainable method for conjugate reduction by continuous hydrogenation. The methodology was initially showcased with a selection of chalcone derivatives and then shown to be extendable to other $\alpha / \beta$-unsaturated carbonyl compounds. In general, high total conversions and excellent chemoselectivities were obtained. A number of hydrogenatively sensitive functionalities such as aryl ketones, benzyl esters and aryl halides were shown to be tolerated well under the reaction conditions. Analysis of the methodology with the metric GreenMotion $^{\mathrm{TM}}$ highlighted a number of key sustainability features, confirming that the approach can be considered green. Work is on-going to up-scale this process (targeting ca. $1 \mathrm{~kg} \mathrm{day}^{-1}$ ) using a custom-built trickle-bed hydrogenation reactor and these studies will be reported in due course.

\section{Conflicts of interest}

The authors declare no conflicts of interest.

\section{Acknowledgments}

This work was funded by INNOVATE UK, Grant No. 104202 and EPSRC (EP/P013341/1). The authors would also like to thank R. Wilson, M. Guyler and R. Meehan from the University of Nottingham for technical assistance.

\section{Notes and references}

(1) Cue, B. W.; Zhang, J. Green Process Chemistry in the Pharmaceutical Industry. Green Chem. Lett. Rev. 2009, 2, 193-211.

(2) Dugger, R. W.; Ragan, J. A.; Ripin, D. H. B. Survey of GMP Bulk Reactions Run in a Research Facility between 1985 and 2002. Org. Process Res. Dev. 2005, 9, 253-258.

(3) Jiménez-González, C.; Poechlauer, P.; Broxterman, Q. B.; Yang, B.-S.; am Ende, D.; Baird, J.; Bertsch, C.; Hannah, R. E.; Dell'Orco, P.; Noorman, H.; et al. Key Green Engineering Research Areas for Sustainable Manufacturing: A Perspective from Pharmaceutical and Fine Chemicals Manufacturers. Org. Process Res. Dev. 2011, 15, 900-911.

(4) Hartman, R. L.; McMullen, J. P.; Jensen, K. F. Deciding Whether To Go with the Flow: Evaluating the Merits of Flow Reactors for Synthesis. Angew. Chemie Int. Ed. 2011, 50, 7502-7519.

(5) Wegner, J.; Ceylan, S.; Kirschning, A. Flow Chemistry - A Key Enabling Technology for (Multistep) Organic Synthesis. Adv. Synth. Catal. 2012, 354, 17-57.

(6) Wiles, C.; Watts, P. Continuous Flow Reactors: A Perspective. Green Chem. 2012, 14, 38-54.

(7) Malet-Sanz, L.; Susanne, F. Continuous Flow Synthesis. A Pharma Perspective. J. Med. Chem. 2012, 55, 40624098.

(8) Baxendale, I. R. The Integration of Flow Reactors into Synthetic Organic Chemistry. J. Chem. Technol. Biotechnol. 2013, 88, 519-552.

(9) Pastre, J. C.; Browne, D. L.; Ley, S. V. Flow Chemistry Syntheses of Natural Products. Chem. Soc. Rev. 2013, 42, 8849-8869.

(10) Kim, H.; Nagaki, A.; Yoshida, J. A Flow-Microreactor Approach to Protecting-Group-Free Synthesis Using Organolithium Compounds. Nat. Commun. 2011, 2, 264.

(11) Alvarez, A. J.; Myerson, A. S. Continuous Plug Flow Crystallization of Pharmaceutical Compounds. Cryst. Growth Des. 2010, 10, 2219-2228.

(12) Deadman, B. J.; Battilocchio, C.; Sliwinski, E.; Ley, S. V. A Prototype Device for Evaporation in Batch and Flow Chemical Processes. Green Chem. 2013, 15, 2050-2055.

(13) Fan, X.; Sans, V.; Yaseneva, P.; Plaza, D. D.; Williams, J.; Lapkin, A. Facile Stoichiometric Reductions in Flow: An Example of Artemisinin. Org. Process Res. Dev. 2012, 16, 1039-1042.

(14) Broom, T.; Hughes, M.; Szczepankiewicz, B. G.; Ace, K.; Hagger, B.; Lacking, G.; Chima, R.; Marchbank, G.; 
Alford, G.; Evans, P.; et al. The Synthesis of Bromomethyltrifluoroborates through Continuous Flow Chemistry. Org. Process Res. Dev. 2014, 18, 1354-1359.

(15) Hessel, V.; Kralisch, D.; Kockmann, N.; Noël, T.; Wang, Q. Novel Process Windows for Enabling, Accelerating, and Uplifting Flow Chemistry. ChemSusChem 2013, 6, 746-789.

(16) Johnson, M. D.; May, S. A.; Calvin, J. R.; Remacle, J.; Stout, J. R.; Diseroad, W. D.; Zaborenko, N.; Haeberle, B. D.; Sun, W.-M.; Miller, M. T.; et al. Development and Scale-Up of a Continuous, High-Pressure, Asymmetric Hydrogenation Reaction, Workup, and Isolation. Org. Process Res. Dev. 2012, 16, 1017-1038.

(17) Lévesque, F.; Seeberger, P. H. Continuous-Flow Synthesis of the Anti-Malaria Drug Artemisinin. Angew. Chemie Int. Ed. 2012, 51, 1706-1709.

(18) Viviano, M.; Glasnov, T. N.; Reichart, B.; Tekautz, G.; Kappe, C. O. A Scalable Two-Step Continuous Flow Synthesis of Nabumetone and Related 4-Aryl-2-Butanones. Org. Process Res. Dev. 2011, 15, 858-870.

(19) Amara, Z.; Streng, E. S.; Skilton, R. A.; Jin, J.; George, M. W.; Poliakoff, M. Automated Serendipity with SelfOptimizing Continuous-Flow Reactors. European J. Org. Chem. 2015, 2015, 6141-6145.

(20) Amara, Z.; Bellamy, J. F. B.; Horvath, R.; Miller, S. J.; Beeby, A.; Burgard, A.; Rossen, K.; Poliakoff, M.; George, M. W. Applying Green Chemistry to the Photochemical Route to Artemisinin. Nat. Chem. 2015, 7, 489-495. Han, X.; Bourne, R. A.; Poliakoff, M.; George, M. W. Immobilised Photosensitisers for Continuous Flow Reactions of Singlet Oxygen in Supercritical Carbon Dioxide. Chem. Sci. 2011, 2, 1059-1067. Reagents. Angew. Chemie Int. Ed. 2014, 53, 3353-3357.

Clark, C. A.; Lee, D. S.; Pickering, S. J.; Poliakoff, M.; George, M. W. UV PhotoVap: Demonstrating How a Simple and Versatile Reactor Based on a Conventional Rotary Evaporator Can Be Used for UV Photochemistry. Org. Process Res. Dev. 2018, 22, 595-599.

Lee, D. S.; Amara, Z.; Clark, C. A.; Xu, Z.; Kakimpa, B.; Morvan, H. P.; Pickering, S. J.; Poliakoff, M.; George, M. W. Continuous Photo-Oxidation in a Vortex Reactor: Efficient Operations Using Air Drawn from the Laboratory. Org. Process Res. Dev. 2017, 21, 1042-1050.

Delaney, E. N.; Lee, D. S.; Elliott, L. D.; Jin, J.; Booker-Milburn, K. I.; Poliakoff, M.; George, M. W. A LaboratoryScale Annular Continuous Flow Reactor for UV Photochemistry Using Excimer Lamps for Discrete Wavelength Excitation and Its Use in a Wavelength Study of a Photodecarboxlyative Cyclisation. Green Chem. 2017, 19, 1431-1438.

(26) Wu, L.; Abada, Z.; Lee, D. S.; Poliakoff, M.; George, M. W. Combining Engineering and Chemistry for the Selective Continuous Production of Four Different Oxygenated Compounds by Photo-Oxidation of Cyclopentadiene Using Liquid and Supercritical CO 2 as Solvents. Tetrahedron 2018, 74, 3107-3112.

(27) Rincón, J. A.; Barberis, M.; González-Esguevillas, M.; Johnson, M. D.; Niemeier, J. K.; Sun, W.-M. Safe, Convenient Ortho- Claisen Thermal Rearrangement Using a Flow Reactor. Org. Process Res. Dev. 2011, 15, 1428-1432.

(28) Baumann, M.; Baxendale, I. R. The Rapid Generation of Isothiocyanates in Flow. Beilstein J. Org. Chem. 2013, 9, 1613-1619.

Newton, S.; Carter, C. F.; Pearson, C. M.; de C. Alves, L.; Lange, H.; Thansandote, P.; Ley, S. V. Accelerating Spirocyclic Polyketide Synthesis Using Flow Chemistry. Angew. Chemie Int. Ed. 2014, 53, 4915-4920. Hartwig, J.; Ceylan, S.; Kupracz, L.; Coutable, L.; Kirschning, A. Heating under High-Frequency Inductive Conditions: Application to the Continuous Synthesis of the Neurolepticum Olanzapine (Zyprexa). Angew. Chemie Int. Ed. 2013, 52, 9813-9817.

Newman, S. G.; Gu, L.; Lesniak, C.; Victor, G.; Meschke, F.; Abahmane, L.; Jensen, K. F. Rapid Wolff-Kishner Reductions in a Silicon Carbide Microreactor. Green Chem. 2014, 16, 176-180.

Nightingale, A. M.; Phillips, T. W.; Bannock, J. H.; de Mello, J. C. Controlled Multistep Synthesis in a ThreePhase Droplet Reactor. Nat. Commun. 2014, 5, 3777.

Sedelmeier, J.; Lima, F.; Litzler, A.; Martin, B.; Venturoni, F. A Multistep Flow Process for the Synthesis of Highly Functionalized Benzoxazoles. Org. Lett. 2013, 15, 5546-5549.

Knudsen, K. R.; Holden, J.; Ley, S. V.; Ladlow, M. Optimisation of Conditions for O-Benzyl and NBenzyloxycarbonyl Protecting Group Removal Using an Automated Flow Hydrogenator. Adv. Synth. Catal. 2007, 349, 535-538.

Ingham, R. J.; Battilocchio, C.; Hawkins, J. M.; Ley, S. V. Integration of Enabling Methods for the Automated Flow Preparation of Piperazine-2-Carboxamide. Beilstein J. Org. Chem 2014, 10, 641-652.

Ouchi, T.; Battilocchio, C.; Hawkins, J. M.; Ley, S. V. Process Intensification for the Continuous Flow Hydrogenation of Ethyl Nicotinate. Org. Process Res. Dev. 2014, 18, 1560-1566.

Lau, S.-H.; Bourne, S. L.; Martin, B.; Schenkel, B.; Penn, G.; Ley, S. V. Synthesis of a Precursor to Sacubitril Using Enabling Technologies. Org. Lett 2015, 17, 5436-5439.

Amara, Z.; Poliakoff, M.; Duque, R.; Geier, D.; Franciò, G.; Gordon, C. M.; Meadows, R. E.; Woodward, R.; 
Leitner, W. Enabling the Scale-Up of a Key Asymmetric Hydrogenation Step in the Synthesis of an API Using Continuous Flow Solid-Supported Catalysis. Org. Process Res. Dev. 2016, 20, 1321-1327.

(39) Han, X.; Poliakoff, M. Continuous Reactions in Supercritical Carbon Dioxide: Problems, Solutions and Possible Ways Forward. Chem. Soc. Rev 2012, 41, 1428-1436.

(40) Stevens, J. G.; Bourne, R. A.; Twigg, M. V.; Poliakoff, M. Real-Time Product Switching Using a Twin Catalyst System for the Hydrogenation of Furfural in Supercritical $\mathrm{CO}_{2}$. Angew. Chemie Int. Ed. 2010, 49, 8856-8859.

(41) Licence, P.; Ke, J.; Sokolova, M.; Ross, S. K.; Poliakoff, M. Chemical Reactions in Supercritical Carbon Dioxide: From Laboratory to Commercial Plant. Green Chem. 2003, 5, 99-104.

(42) Bourne, R. A.; Stevens, J. G.; Ke, J.; Poliakoff, M. Maximising Opportunities in Supercritical Chemistry: The Continuous Conversion of Levulinic Acid to $\mathrm{Y}$-Valerolactone in $\mathrm{CO}_{2}$. Chem. Commun. 2007, 4632-4634.

(43) Rivière, C. Dihydrochalcones. In Studies in Natural Products Chemistry; Elsevier, 2016; pp 253-381.

(44) Perez Gutierrez, R. M.; Muñiz-Ramirez, A.; Valdes Sauceda, J. African Journal of Pharmacy and Pharmacology Review: The Potential of Chalcones as a Source of Drugs. 2015, 9, 237-257.

(45) Zhou, B.; Xing, C. Diverse Molecular Targets for Chalcones with Varied Bioactivities. Med. Chem. (Los. Angeles). 2015, 5, 388-404.

(46) Gomes, M.; Muratov, E.; Pereira, M.; Peixoto, J.; Rosseto, L.; Cravo, P.; Andrade, C.; Neves, B. Chalcone Derivatives: Promising Starting Points for Drug Design. Molecules 2017, 22, 1210.

(47) Mahapatra, D. K.; Bharti, S. K.; Asati, V. Chalcone Scaffolds as Anti-Infective Agents: Structural and Molecular Target Perspectives. Eur. J. Med. Chem. 2015, 101, 496-524.

(48) Hattori, K.; Sajiki, H.; Hirota, K. Chemoselective Control of Hydrogenation among Aromatic Carbonyl and Benzyl Alcohol Derivatives Using Pd/C(En) Catalyst. Tetrahedron 2001, 57, 4817-4824.

(49) Rylander, P. N. Hydrogenation Methods; Academic: New York, 1985.

(50) Hudlicky, M. Reductions in Organic Chemistry, 2nd ed.; ACS: Washington, DC, 1996.

(51) Lipshutz, B. Rediscovering Organocopper Chemistry Through Copper Hydride. It's All About the Ligand. Synlett 2009, 4, 509-524.

(52) Deutsch, C.; Krause, N.; Lipshutz, B. H. CuH-Catalyzed Reactions. Chem. Rev. 2008, 108, 2916-2927.

(53) Lipshutz, B. H.; Servesko, J. M.; Petersen, T. B.; Papa, P. P.; Lover, A. A. Asymmetric 1,4-Reductions of Hindered $\beta$-Substituted Cycloalkenones Using Catalytic SEGPHOS-Ligated CuH. Org. Lett. 2004, 6, 12731275.

(54) Appella, D. H.; Moritani, Y.; Shintani, R.; Ferreira, E. M.; Buchwald, S. L. Asymmetric Conjugate Reduction of $\alpha, \beta$-Unsaturated Esters Using a Chiral Phosphine - Copper Catalyst. J. Am. Chem. Soc. 1999, 121, 9473-9474.

(55) Zhou, Y.; Bandar, J. S.; Liu, R. Y.; Buchwald, S. L. CuH-Catalyzed Asymmetric Reduction of $\alpha, \beta$-Unsaturated Carboxylic Acids to $\beta$-Chiral Aldehydes. J. Am. Chem. Soc. 2018, 140, 606-609.

(56) Zhang, L.; Winterbottom, J. M.; Boyes, A. P.; Raymahasay, S. Studies on the Hydrogenation of Cinnamaldehyde over Pd/C Catalysts. J. Chem. Technol. Biotechnol. 1998, 72, 264-272.

(57) Kobayashi, J.; Mori, Y.; Okamoto, K.; Akiyama, R.; Ueno, M.; Kitamori, T.; Kobayashi, S. A Microfluidic Device for Conducting Gas-Liquid-Solid Hydrogenation Reactions. Science 2004, 304, 1305-1308.

(58) Rathi, A. K.; Gawande, M. B.; Ranc, V.; Pechousek, J.; Petr, M.; Cepe, K.; Varma, R. S.; Zboril, R. Continuous Flow Hydrogenation of Nitroarenes, Azides and Alkenes Using Maghemite-Pd Nanocomposites. Catal. Sci. Technol. 2016, 6, 152-160.

(59) Sullivan, R. J.; Newman, S. G. Chiral Auxiliary Recycling in Continuous Flow: Automated Recovery and Reuse of Oppolzer's Sultam. Chem. Sci. 2018, 9, 2130-2134.

(60) Nagendiran, A.; Sörensen, H.; Johansson, M. J.; Tai, C.-W.; Bäckvall, J.-E. Nanopalladium-Catalyzed Conjugate Reduction of Michael Acceptors - Application in Flow. Green Chem. 2016, 18, 2632-2637.

(61) Hsieh, C.-T.; Ötvös, S. B.; Wu, Y.-C.; Mándity, I. M.; Chang, F.-R.; Fülöp, F. Highly Selective Continuous-Flow Synthesis of Potentially Bioactive Deuterated Chalcone Derivatives. Chempluschem 2015, 80, 859-864.

(62) Mori, A.; Miyakawa, Y.; Ohashi, E.; Haga, T.; Maegawa, T.; Sajiki, H. Pd/C-Catalyzed Chemoselective Hydrogenation in the Presence of Diphenylsulfide. Org. Lett. 2006, 8, 3279-3281.

(63) Mori, A.; Mizusaki, T.; Miyakawa, Y.; Ohashi, E.; Haga, T.; Maegawa, T.; Monguchi, Y.; Sajiki, H. Chemoselective Hydrogenation Method Catalyzed by Pd/C Using Diphenylsulfide as a Reasonable Catalyst Poison. Tetrahedron 2006, 62, 11925-11932.

(64) Mori, A.; Mizusaki, T.; Kawase, M.; Maegawa, T.; Monguchi, Y.; Takao, S.; Takagi, Y.; Sajiki, H. Novel Palladium-on-Carbon/Diphenyl Sulfide Complex for Chemoselective Hydrogenation: Preparation, Characterization, and Application. Adv. Synth. Catal. 2008, 350, 406-410.

(65) Mccue, A. J.; Anderson, J. A. Sulfur as a Catalyst Promoter or Selectivity Modifier in Heterogeneous Catalysis. Catal. Sci. Technol. 2014, 4, 272-294.

(66) Su, C.; Li, X.-N.; Zhang, Q.-F.; Ma, L.; Lu, C.-S.; Feng, F. Behavior of Adsorbed Diphenyl-Sulfide on the Pd/C Catalyst for o-Chloronitrobenzene Hydrogenation. Chinese Chem. Lett. 2013, 24, 59-62. 
(67) Jackson, M. A.; Appell, M.; Blackburn, J. A. Hydrodeoxygenation of Fructose to 2,5-Dimethyltetrahydrofuran Using a Sulfur Poisoned Pt/C Catalyst. Ind. Eng. Chem. Res. 2015, 54, 7059-7066.

(68) Yun, S.; Lee, S.; Yook, S.; Patel, H. A.; Yavuz, C. T.; Choi, M. Cross-Linked "Poisonous" Polymer: Thermochemically Stable Catalyst Support for Tuning Chemoselectivity. ACS Catal. 2016, 6, 2435-2442.

(69) Mckenna, F.-M.; Wells, R. P. K.; Anderson, J. A. Enhanced Selectivity in Acetylene Hydrogenation by Ligand Modified Pd/TiO2 Catalysts. Chem. Commun 2011, 47, 2351-2353.

(70) Makosch, M.; Lin, W.-I.; Bumbaíek, V.; Sá, J.; Medlin, J. W.; Hungerbühler, K.; Van Bokhoven, J. A. Organic Thiol Modified Pt/TiO2 Catalysts to Control Chemoselective Hydrogenation of Substituted Nitroarenes. ACS Catal. 2012, 2, 2079-2081.

(71) Peng, F.; Du, Q.; Peng, C.; Wang, N.; Tang, H.; Xie, X.; Shen, J.; Chen, J. A Review: The Pharmacology of Isoliquiritigenin. Phyther. Res. 2015, 29, 969-977.

(72) Gutmann, A.; Bungaruang, L.; Weber, H.; Leypold, M.; Breinbauer, R.; Nidetzky, B. Towards the Synthesis of Glycosylated Dihydrochalcone Natural Products Using Glycosyltransferase-Catalysed Cascade Reactions. Green Chem. 2014, 16, 4417-4425.

(73) Phan, T. V. T.; Gallardo, C.; Mane, J. GREEN MOTION: A New and Easy to Use Green Chemistry Metric from Laboratories to Industry. Green Chem. 2015, 17, 2846-2852.

(74) Anastas, P.; Eghbali, N. Green Chemistry: Principles and Practice. Chem. Soc. Rev. 2010, 39, 301-312.

(75) Roschangar, F.; Sheldon, R. A.; Senanayake, C. H. Overcoming Barriers to Green Chemistry in the Pharmaceutical Industry - the Green Aspiration Level ${ }^{\mathrm{TM}}$ Concept. Green Chem. 2015, 17, 752-768.

(76) Trost, B. M. The Atom Economy - A Search for Synthetic Efficiency. Science 1991, 254, 1471-1477.

\section{For table of contents use only:}

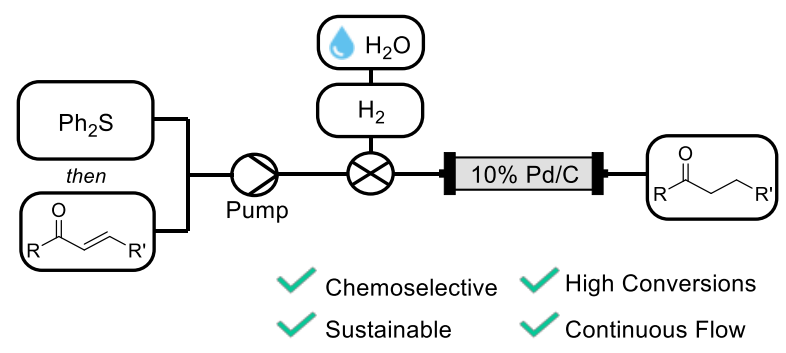

